ÉGYPTE

monde arabe

\section{Égypte/Monde arabe}

14 | 1993

Dits et écrits, mémoires et rites

\title{
Le tremblement de terre en Égypte
}

Analyse de presse

\section{Galila El Kadi}

\section{(2) OpenEdition}

Journals

Édition électronique

URL : https://journals.openedition.org/ema/584

DOI : 10.4000/ema.584

ISSN : 2090-7273

Éditeur

CEDEJ - Centre d'études et de documentation économiques juridiques et sociales

Édition imprimée

Date de publication : 30 juin 1993

Pagination : 163-196

ISSN : 1110-5097

Référence électronique

Galila El Kadi, «Le tremblement de terre en Égypte », Égypte/Monde arabe [En ligne], 14 | 1993, mis en ligne le 08 juillet 2008, consulté le 07 juillet 2022. URL : http://journals.openedition.org/ema/584 ; DOI : https://doi.org/10.4000/ema.584

Ce document a été généré automatiquement le 7 juillet 2022.

Tous droits réservés 


\title{
Le tremblement de terre en Égypte
}

\author{
Analyse de presse
}

\section{Galila El Kadi}

1 Sentir la terre trembler sous ses pieds n'est pas un phénomène courant dans la vallée du Nil. C'est pourtant ce qui s'est produit le 12 octobre 1992. Le séisme qui a secoué Masr al-Mahrûsa pendant près d'une minute (59 secondes exactement) avait pour épicentre le mont Qatrani, dans la région du Fayoum, à $80 \mathrm{~km}$ au sud-ouest du Caire. D'une amplitude moyenne (5,6 sur l'échelle de Richter), la secousse a néanmoins causé des dégâts considérables: 561 morts, 9.929 blessés, 5.000 immeubles complètement effondrés, 115.000 constructions déclarées hors d'usage, 2.748 écoles présentant des fissures plus ou moins importantes, 187 monuments historiques endommagés.

2 Parmi les 26 gouvernorats qui constituent le territoire égyptien, 8 ont été touchés de façon inégale par le séisme, les plus durement affectés étant ceux du Caire, de Giza et du Fayoum.

3 Cette secousse d'une minute a suscité une vive polémique qui a occupé la « une » de la presse pendant plus de deux mois. Dans la mesure où de tels cataclysmes sont le fait de la nature, il semblait difficile d'en faire assumer la responsabilité directe au gouvernement... Néanmoins, le manque de prévoyance de celui-ci en amont du séisme et, en aval, sa capacité à gérer la crise ont été au centre des débats.

4 Exclus des dispositifs d'intervention en faveur des sinistrés, les partis de l'opposition ont intensifié leurs critiques contre le gouvernement et le Parti national, qui ont monopolisé à tous les niveaux le droit d'agir sans en avoir les moyens et sans faire preuve d'une grande efficacité. Chaque parti a ainsi présenté sa conception des événements et de leurs conséquences: pour les libéraux du Néo-Wafd, ennemis farouches de la Révolution de Juillet et de ses réalisations, c'est le Haut-Barrage qui a été d'emblée rendu responsable du séisme, induit selon eux par le poids de l'eau du lacréservoir Nasser. Le Parti du rassemblement progressiste, quant à lui, a surtout critiqué les contradictions entre le discours officiel (relatif notamment à la nature et au volume de l'aide apportée aux sinistrés) et les faits, soulignant la violation des règlements de construction et la non-conformité des matériaux utilisés, et dénonçant les conditions de vie des pauvres, les plus nombreux à pâtir du séisme. Ainsi politisé, le séisme s'est 
également "islamisé », puisque le Parti du travail (de tendance islamiste) y a vu le signe de la colère divine s'abattant sur un gouvernement corrompu et une société éloignée du droit chemin.

5 Répondant à ses détracteurs, le Parti national au pouvoir a contre-attaqué par la voie de sa presse officielle, dénonçant tant les surenchères politiciennes à visées purement électorales que les abus commis par certains sinistrés, abus justifiant aux yeux du pouvoir le contrôle de toutes les interventions, y compris la collecte et l'affectation des dons.

En dépit de ces divergences, certains thèmes majeurs ont cependant fait l'unanimité : l'hypercentralisation de l'Etat, l'absence de système de gestion des crises, la fragilité des constructions due à la violation des normes de construction et d'urbanisme, la gestion défectueuse du patrimoine, la concentration urbaine, les politiques d'aménagement inadéquates.

Les premières réactions

7 «Un tremblement de terre destructeur a secoué l'Égypte pendant soixante secondes »; « 368 morts, 3.500 blessés, effondrement ou fissures de 182 bâtiments dans tous les gouvernorats de l'Égypte » (al-Ahrâm, 13/10) ; «Le nombre de morts et de blessés est le double des chiffres officiels : 800 morts selon les agences de presse » (al-Cha'b, 13/10); « 500 morts, 4.000 blessés et des centaines de victimes toujours sous les décombres » (al-Wafd,14/10).

8 «Le tremblement de terre, d'une amplitude de 6 sur l'échelle de Richter, a duré 2 minutes dans le gouvernorat de Charqiyya et y a causé d'importants dégâts » (al-Cha'b, $13 / 10)$; « Le gouvemorat de Charqiyya à subi très peu de dégâts ; aucun effondrement de bâtiment public n'a été signalé, le bilan s'élève à 3 morts et 8 blessés » (déclarations du gouverneur de Charqiyya, al-Ahrâm, 13/10).

9 «Sept citoyens sautent par les fenêtres et depuis les toits au moment du séisme » (alAhâli, 14/10); "Les élèves des écoles d'Imbaba se sont sauvés en sautant par les fenêtres : 20 morts et 29 blessés "; "Les écoliers sont les principales victimes du tremblement de terre»; " 191 écoliers blessés et 12 morts dans le gouvernerai de Gharbiyya »; " 12 élèves piétinés à mort par leurs camarades dans une école à Helwan »; «Effondrement de 6 écoles préparatoires et primaires à Helwan » (al-Ahrâm, $13 / 10 ;$ al-Wafd, 14/10).

10 "L'évacuation d'écoles fissurées une semaine avant le séisme nous a évité une catastrophe " (le gouverneur de Charqiyya à al-Ahrâm, 13/10); « 8 écoliers victimes de la cohue dans une école à Alexandrie »; "La cohue a doublé le nombre des victimes, elle a souvent provoqué des effondrements » (al-Ahrâm, 13/10);

11 «Sayyida Zaynab et Darb al-Ahmar; les quartiers les plus touchés par le séisme » (alWafd, 14/10) ; «Les maisons qui se sont effondrées dans le quartier de Ma'ràf étaient depuis longtemps déclarées hors d'usage, mais le gouvernerai n'a pas fait le nécessaire pour le relogement des habitants » (al-Ahali, 14/10).

12 «1.100 appartements pour le relogement des ménages sinistrés »; «Le président ordonne que l'aide aux familles des sinistrés soit versée sans délai » (al-Ahrâm, 13/10); " 30 unités d'habitations d'urgence pour reloger les sans-abri à Assiout» (al-Ahâli, 14/10) ; « 80 maisons partiellement ou totalement effondrées dans la vieille ville; la plupart étaient impropres à l'habitation »; "Que Dieu est clément ! Si le séisme avait duré 60 secondes de plus, la moitié du Caire serait détruite » (al-Cha'b, 13/10). 
« 70 millions \$ offerts par l'Arabie Saoudite et le Koweït »; "Pour la première fois depuis la guerre de 73, les dons de sang dépassent largement les besoins. » (al-Cha'b, 13/10) "Les sans-abri campent dans les rues et sur les places" (al-Wafd, 14/10); "Craignant une nouvelle secousse, des centaines de citoyens ont passé la nuit dans les jardins publics " (al-Ahâli, 14/10) : «Les propriétaires de résidences secondaires à Alexandrie ont fui la capitale suite à l'annonce de la fermeture des écoles; Les routes menant à la deuxième ville d'Égypte ont subi des encombrements inhabituels. " (alWafd, 14/10)

14 «S'appuyant sur les données fournies par la station sismographique de Helwan, le docteur Joseph Sidqi, président de l'Institut national de Recherche spatiale, géophysique et géologique, assure aux citoyens que l'éventualité d'un tremblement de terre de la même violence est exclue.» (...) «Les équipements de la station de Helwan sont obsolètes, incapables de mesurer un séisme d'une amplitude inférieure à 4 sur l'échelle de Richter ; par conséquent, ils ne peuvent émettre aucune prévision. » (alAhrâm, 13/10) «L'Égypte a subi en 1969 et en 1974 deux séismes de même amplitude, dont les épicentres se situaient respectivement en mer Rouge et à Abu Hammad (Charqiyya); ce genre de secousses peut se reproduire à l'avenir. » (al-Ahrâm, 13/10) « Le grand sismologue Ruchdi Saïd, émigré aux Etats-Unis, a déclaré à al-Ahâli, au cours d'un entretien téléphonique, que cette secousse est la plus forte qu'ait connu l'Égypte depuis un siècle. » (al-Ahâli 14/10)

15 «Le ministre de l'Information a déclaré que tous les sinistrés avaient été inventoriés dans l'ensemble des gouvernorats, que les forces de la défense civile, épaulées par celles du Ministère de l'Intérieur et de la police, assumaient leur rôle à tous les niveaux en secourant les blessés et en relogeant les sans-abri, et que le Ministère des Affaires sociales se chargeait de verser des indemnités d'urgence aux sinistrés. Le ministre a conclu en assurant qu'aucun monument historique n'avait été touché par le séisme.» (...) «Effondrement de la mosquée al-Tachtuchi à Bab al-Cha'riyya » (al-Ahrâm,13/10); "Chute des croissants surmontant les minarets de la mosquée d'al-Azhar » (al-Cha'b 13/10) ; «L'Organisme des Antiquités lance un appel au Ministère du Développement et de l'Habitat pour sauver les monuments islamiques... Affaissement des minarets de Ghuriyya et de Gamaliyya et fissures dans les coupoles des mosquées d'al-Ghuri et de Qalaoun » (al-Ahâli, 14/10).

16 "Les initiatives populaires ont comblé le manque de moyens officiels... Un grand nombre de citoyens se sont portés volontaires pour transporter les blessés dans les hôpitaux et héberger les sans-abri. » (...) « Trois organismes sont chargés par le premier ministre de faire l'inventaire des morts et des blessés, d'où d'importants écarts d'estimation. " (al-Ahâli, 14/10) "Atif Sidqi préside un comité de 10 ministres pour suivre la situation »; " Le président Moubarak a chargé tous les ministères mis en état d'urgence de prendre 30 mesures d'urgence pour secourir les sinistrés en passant outre aux démarches bureaucratiques. » (al-Ahrâm, 13/10) « La France, l'Angleterre, la Suisse et l'Allemagne proposent d'envoyer des équipes de secours pour aider l'Égypte.» (...) «Un immeuble de 17 étages s'effondre comme un château de cartes en quelques secondes à Héliopolis. » (...) «Le président Moubarak interrompt sa visite en Chine et rentre en Égypte ce soir... » (al-Ahrâm, 13/10). '

17 . Ces quelques titres et commentaires sélectionnés dans la presse le lendemain du «Lundi noir » ou du «Lundi de la terreur » témoignent de l'épouvante, de l'angoisse et surtout de la confusion qui s'est emparée de tous, gouvernants comme gouvernés. Les 
contradictions et l'imprécision des informations diffusées par la presse officielle sur les causes du séisme, sa nature, ses antécédents, l'importance des dégâts et le nombre des victimes n'étaient pas de nature à calmer les esprits. Commentant par la suite ces premières réactions, un journaliste écrit : «II est étonnant de constater que dans un pays qui possède dix mille docteurs, il n'y ait pas un seul spécialiste capable d'expliquer simplement à la population quelles forces provoquent un tremblement de terre et quelle est la signification de l'échelle de Richter.» (October, 16/10) Ce manque de « transparence » a donné lieu à toutes sortes d'extrapolations. Les uns ont exagérément grossi l'événement, le qualifiant du « plus grand tremblement de terre que l'Égypte ait jamais subi dans son histoire » ou affirmant que l'Égypte était entrée « dans la ceinture des séismes ", tandis que d'autres en minimisaient les effets en banalisant les dégâts et en assurant qu'il n'y aurait pas d'autres secousses. Or, plusieurs secousses de moindre intensité ont été ressenties par les Cairotes deux heures après la secousse principale. L'on s'est contenté de les signaler sans expliquer que de telles « répliques » peuvent se produire pendant plusieurs semaines, voire plusieurs mois, à la suite d'un séisme. Sur le terrain, la lenteur des secours et leur insuffisance, l'inefficacité ou l'absence de moyens, le manque de coordination entre les organismes intervenants ont ajouté à la panique et à la confusion générales.

18 Au cours des quatre jours suivant le séisme, deux événements « majeurs » feront la une de la presse : l'interruption du voyage du président Moubarak et l'effondrement, à Héliopolis, d'un immeuble résidentiel de construction récente.

Polarisation urbaine et hypercentralité de l'Etat

19 Construit il y a à peine dix ans, l'immeuble d'Héliopolis s'est transformé en un amoncellement de gravats, ensevelissant sous ses décombres 74 victimes. La concentration d'un aussi grand nombre de morts en un seul lieu, si elle permet de parler d'hécatombe, ne justifie cependant pas l'intérêt démesuré accordé à un seul des 102 édifices qui se sont effondrés dans la capitale. Quant aux villages entièrement détruits dans les gouvernorats de Giza et du Fayoum, proches de l'épicentre, on ignorait jusqu'à leur existence. Dès le début du séisme, l'on ne s'est préoccupe que du Caire, capitale et siège du pouvoir, regroupant près du quart de la population du pays. L'intérêt accordé au Caire était donc à la mesure de son importance politique et de son poids démographique.

20 Le déséquilibre de la structure urbaine a tout naturellement engendré un déséquilibre dans le traitement et la diffusion de l'information. Ainsi, la concentration urbaine s'est traduite par une polarisation des moyens mis en œuvre pour secourir les sinistrés et reloger les sans-abri : 3.500 Cairotes relogés en urgence dès les premiers Jours à Qatamiyya. De la même façon, à une autre échelle, un seul quartier, lieu de résidence du président et de $54 \%$ des catégories aisées de la capitale, a polarisé une grande part des efforts locaux et internationaux. Les cinq équipes de secours française, allemande, grecque, algérienne et yougoslave, épaulées par des volontaires venus de Turquie et du Pakistan, n'ont ainsi connu du Caire que le site de l'immeuble sinistré d'Héliopolis! Pendant qu'ils déblayaient les décombres, à la recherche de survivants et exhumaient les corps des victimes avec leurs équipements de pointe, « un habitant du quartier d'alKhalifa était resté perché, isolé pendant quatre jours sans aucun secours, sur le balcon du quatrième étage de son immeuble, suite à l'effondrement de la cage d'escalier. » (alAhrâm, 16/10) A al-'Utuf (Gamaliyya), des familles entières se trouvèrent pendant plus de 12 heures dans la même situation (Sabâh al-Khayr, 29/10). Visitant le quartier de 
Gamaliyya 17 jours après le séisme, une journaliste constate avec étonnement: "Comment croire que Gamaliyya, quartier des monuments historiques, des ateliers, où $70 \%$ des constructions sont vétustes, n'ait vu débarquer aucune équipe de secours jusqu'à maintenant? A la suite de la première secousse, 15 immeubles s'y sont effondrés et 203 fissurés, mais il n'y a eu que deux blessés, car les habitants de ce quartier avaient l'expérience des effondrements d'immeubles bien avant le séisme... Cela leur a permis de prendre des mesures qui ont limité le nombre des victimes. » (Rose al-Yussuf, 29/10). L'intérêt accordé à l'immeuble d'Héliopolis frisait parfois la provocation : des pages entières étaient consacrées aux biographies des 74 victimes et des 24 survivants, photos à l'appui, de leur naissance à leur ensevelissement sous les décombres pour les premiers, ou au moment de leur sauvetage pour les autres. Les objets les plus insignifiants, trouvés dans les gravats, étaient exposés et minutieusement décrits. Lorsque le dernier des survivants fut dégagé sain et sauf après avoir passé 74 heures sous les décombres, le «miracle» d'Héliopolis (ou «Masr alGadida », le " Nouveau Caire ») eut pour effet de focaliser davantage encore l'attention des médias, suscitant l'amertume des laissés-pour-compte de Masr al-Qadima (le «Vieux Caire ») qui «adjuraient les responsables des quartiers de leur rendre visite » (al-Ahrâm, 16/10).

21 Mais la pratique de deux poids, deux mesures, tant pour la rapidité et la qualité des secours que pour l'abondance et la diffusion des informations, entre Héliopolis et les autres quartiers de la capitale, est encore plus flagrante entre cette dernière et les autres gouvernorats :

"Cette pratique héritée du passé devrait être remise en cause par des faits concrets et non par des slogans. Autrefois, le moindre problème affectant la capitale - la pénurie de bouteilles de propane par exemple - suffisait pour que tous les responsables se mobilisent ; la nouvelle se répandait jusqu'aux villages les plus reculés... Inversement, lorsque Qena était inondée par des pluies torrentielles, la nouvelle glissait dans les pages internes des journaux... quand elle n'était pas purement et simplement passée sous silence... Ce mépris pour les ruraux, considérés comme une race inférieure, demeure encore vivace, il continue à commander notre attitude... L'expérience du séisme l'a bien montré... Or le séisme n'a pas fait de différence entre al-Ayat (district de Giza) et le Caire, qui furent affectés de la même façon. Cependant les regards se sont concentrés sur le Caire et, jusqu'au 18 octobre, il n'a jamais été question d'al-Ayat. C'est seulement grâce au journal d'opposition al-Wafd qu'on a découvert l'existence de lieux sinistrés ailleurs qu'au Caire. » (al-Wafd, 17/11). Signalons toutefois une information sur al-Ayat dans al-Ahrâm du 16/10: «En 72 heures, les organes du Ministère des Transports et de l'organisme général des Ponts et Chaussée ont achevé de réparer la route reliant al-Ayat à al-Maraziq, qui.s'est affaissée à deux endroits, respectivement de $70 \mathrm{~cm}$ de profondeur sur 1,50 m de long et de 1,50 m de profondeur sur $70 \mathrm{~m}$ de long. » Sous le titre : "Loin des lumières du Caire ", un Journaliste écrit : "Bemest, Bamich, Badsa, Mazghuta... ne sont pas les noms de villes étrangères ou de villages où il fait bon vivre... Il s'agit de quatre villages appartenant à Markaz al-Ayat (Giza), vivant audessous du seuil de pauvreté. Le séisme a ajouté à leurs malheurs la destruction. En quelques secondes, 280.000 personnes se sont retrouvées sans abri, 53 autres ont été ensevelies sous les décombres et sont venues s'ajouter aux 180 morts recensés dans le gouvernorat de Giza. » (al-Wafd, 18/10) 
Plus loin encore des lumières du Caire, les villages de Tamya (région du Fayoum) n'ont été mentionnés dans la presse qu'à partir du 26 alors que, situés à $10 \mathrm{~km}$ de l'épicentre, ils furent les premiers à être frappés par le zilzâl... Bilan: " 5 villages entièrement détruits et transformés en un amas de boue et de paille, 28 écoles et 32 bâtiments publics fissurés, près d'un millier de maisons écroulées et 95 endommagées... » (Rose alYoussef, 26/10)

Plus grave encore que l'absence d'informations est l'incurie de la gestion et l'ignorance, par les collectivités locales, du drame de ces citoyens oubliés. On y reviendra.

Le rôle déterminant du chef de l'Etat

«Autant j'ai été touché par le retour précipité du président, autant j'ai déploré l'inexistence d'un système de gestion capable de contenir la crise en faisant des choix pertinents, même en l'absence du chef de l'Etat. Dès son arrivée, Hosni Moubarak a pris les choses en main en plaçant le gouvernement et le Parti national devant leurs responsabilités. Tous les dons en nature devaient parvenir au Croissant Rouge sous la surveillance de Mme Moubarak. Que signifie tout ceci? Tout simplement que le retour du président n'était pas motivé par le simple sentiment de solidarité d'un chef d'Etat vis-à-vis de ses compatriotes dans un moment de crise, mais que son retour était bel et bien indispensable, car il est le maestro... Cet état de fait, qu'on a baptisé « l'orphelinat populaire ", prend fin dès que le chef de l'Etat fait son apparition. En son absence règnent l'effroi et la confusion. Ce qui est devenu évident, c'est que le gouvernement et le Parti ne jouissent d'aucune confiance... Le premier reste paralysé en attendant les ordres, car il n'a pas l'habitude d'affronter seul les crises. Au sein du second, ce sont les initiatives individuelles qui prévalent, sans coordination aucune... » (M. Fawzi, Rose alYoussef, 26/10)

6 "II était impossible, en l'absence du chef de l'Etal, de prendre des mesures d'exception pour réparer les dégâts causés par le séisme: lui seul est doté des prérogatives nécessaires. (...) Disons les choses clairement; le retour du chef de l'Etat a accéléré la marche des choses et a contribué à atténuer les souffrances des sinistrés... » (G. Selim, al-Wafd, 21/11)

\section{Ou encore:}

«La nature bureaucratique des institutions étatiques leur enlève toute faculté d'initiative, toute capacité d'imagination et tout pouvoir décisionnel pour affronter les cataclysmes. (...) La centralité de la fonction présidentielle se manifeste par la paralysie, la confusion, voire l'absence du gouvernement, évidente, à la veille du retour du chef de l'Etat. » (Kamal al-Said, al-Wafd, 7/11)

29 Ces trois commentaires sur le rôle central du chef de l'Etat montrent que la décentralisation administrative et la démocratisation de la vie politique mises en œuvre depuis 1973 ne se sont pas traduites par une réelle autonomie de gestion et de décision au niveau des différentes institutions étatiques et des collectivités locales. Le problème est moins celui de la bureaucratie que celui des rapports paternalistes pesants qui caractérisent la société égyptienne, réalité amplifiée par les médias (on a pu voir de multiples photos représentant Hosni Moubarak en «bon papa » rendant visite à ses « enfants » dans les hôpitaux et les lieux sinistrés).

Gestion de la crise et crise de la gestion

Dès avant le retour du chef de l'Etat, et conformément aux 30 directives dictées par celui-ci à bord de son avion, le gouvernement a pris les mesures nécessaires pour parer 
au plus pressé : il a d'abord décrété l'état d'urgence dans tous les hôpitaux, a dépêché des secours en fonction des moyens disponibles sur les lieux du sinistre, en particulier dans la capitale, et s'est assuré de l'état des infrastructures de base et des équipements d'importance stratégique tels que le Haut-Barrage, les centrales électriques, les stations pétrolières... Il a envoyé des avions repérer les lieux affectés par le séisme, a pris la décision de verser 500 livres égyptiennes à chaque famille ayant perdu son logement ou son tuteur. Mais l'ampleur des dégâts et le manque de coordination entre le pouvoir central et les collectivités locales d'une part, et entre celui-ci et les différents organismes œuvrant sur le terrain, d'autre part, conjugués au manque d'effectifs dans une situation qui exigeait des moyens exceptionnels, se sont souvent traduits par des lenteurs générant des situations insupportables. L'absence de coordination a donné lieu à la multiplication des initiatives individuelles ou, à l'inverse, à l'inertie totale.

31 . Dès son arrivée, le chef de l'Etat s'est rendu d'abord sur le site de l'immeuble d'Héliopolis ; il a ensuite réuni le Conseil des ministres et défini un programme d'action où le rôle de chacun était clairement déterminé :

32 - les gouvernorats étaient chargés de faire l'inventaire des bâtiments endommagés et d'effectuer les expertises techniques dans un délai d'une semaine afin de déterminer le nombre d'unités de relogement requises. Ils devaient recourir aux ingénieurs de l'armée et des universités pour renforcer leurs effectifs.

Ils étaient également chargés d'établir une liste précise des unités d'habitation qui présentaient déjà un danger avant le séisme étant donné leur vétusté, et d'adresser cette liste au Premier ministre.

Ils devaient en outre trouver les unités de relogement requises parmi celles réalisées dans le cadre du second plan quinquennal, soit par les gouvernorats eux-mêmes, soit par le Ministère du Développement et de l'Habitat, le conseil des ministres se chargeant d'affecter les sommes nécessaires à la réalisation d'infrastructures de base et aux finitions; les grandes entreprises étaient chargées par le premier ministre d'effectuer ces travaux dans les délais définis au préalable.

35 - Aide aux sinistrés :

36 - Augmentation de l'indemnité de dédommagement (de 500 à 5.000 LE si décès du chef de famille ou perte du logement, et de 200 à 1.000 LE pour les blessés.

37 - Aménagement de zones temporaires de relogement dans les centres d'accueil, l'armée se chargeant de dresser des tentes et l'Etat prenant en charge l'hébergement et la nourriture.

- Prise en charge par l'Etat de la restauration des bâtiments endommagés.

39 Dons:

40 - Deux comptes sous contrôle de l'Etat ont été ouverts; les dons en nature devaient parvenir au Croissant Rouge sous le contrôle de Mme Moubarak.

41 Un foisonnement de déclarations prometteuses a accompagné ces mesures, donnant ainsi l'espoir aux sinistrés que l'Etat-Providence allait effacer en quelques jours toutes les conséquences du séisme :

42 "L'Etat ne laissera pas un seul citoyen dans la rue », déclare le premier ministre (alAhrâm, 16/10) ; « 56.500 logements pour les sinistrés 》 (al-Ahrâm, 16/10) ; «1.000 logements au Caire et 100 logements à Giza prêts à accueillir les sans-abri » (al-Ahrâm, al-Akhbâr, 13 et 16/10); «Le Ministère de l'Habitat répondra à toutes les demandes 
d'unités de relogement provenant de tous les gouvernorats » (al-Akhbâr, 14/10) ; «Le ministre de l'Administration locale a déclaré qu'il existait 30.000 logements vacants, parmi lesquels 7.000 partiellement équipés et les 23.000 restants, situés dans le new settlment d'al-Nahda, non équipés. Le ministre a ajouté que 10 millions LE seraient alloués à l'équipement de 700 logements à Giza et 800 à Qaliubiyya. Simultanément, le président de l'Assemblée du Peuple, par ailleurs député de la circonscription de Sayyida Zaynab, a déclaré qu'il trouverait des logements à Madinat al-Salam, à l'est du Caire, pour les sinistrés de ce quartier.» (al-Ahrâm, 16/10) "106 comités techniques regroupant 7.000 ingénieurs achèvent d'expertiser les constructions » (al-Ahrâm, 17/10).

43 "Le gouverneur du Caire annonce la formation immédiate de comités pour faire l'inventaire des immeubles effondrés. Le gouvernerai doit également procéder à l'installation, dans un délai de dix jours, d'équipements et services pour 2.000 logements... Les ménages de 5 personnes auront droit à un appartement de deux chambres plus un séjour, les ménages de taille supérieure obtiendront trois chambres et un séjour. » (al-Ahrâm, 16/10) "Selon le secrétaire général du gouvernorat du Caire, 3.000 logements sont disponibles à al-Salam, ce qui dépasse largement les besoins des sans-abri. » (al-Akhbar. 14/10) «Attribution, par le Ministère de l'Enseignement, de la somme de $500 \mathrm{LE}$ à chaque écolier ayant été blessé lors du séisme (...) Attribution de 1.000 LE, décidée par le président de l'Assemblée du Peuple, à chaque famille de Sayyida Zaynab comptant un défunt parmi les siens (...) Le Ministère des Affaires sociales offre des aides aux sinistrés (500 LE pour chaque famille où une personne est décédée, $200 \mathrm{LE}$ pour chaque blessé) et se charge en outre d'héberger 1.000 familles à Madinat al-Salam et 400 à Giza (...) Le ministère va commencer à verser les indemnités exceptionnelles de retraite aux ayants droit (...) 100 LE pour la famille de chaque défunt au Fayoum, déclare le gouverneur (...) Mme Moubarak affirme que le plan dressé par la Croix-Rouge permet d'assurer les besoins de tous les sinistrés » (al-Ahrâm et al-Akhbâr 14/10, alAkhbâr 15/10, al-Ahrâm 17 et 18/10, divers organes officiels du 17/10).

Tournant en dérision ces surenchères, un journaliste d'al-Cha'b écrivait: "Depuis le début du séisme, les promesses affluent sans interruption; un hôpital pour chaque citoyen, un appartement pour chaque sinistré, de la nourriture pour chaque affamé, des vêtements pour chaque nécessiteux.... » (al-Cha'b, 20/10) En fait, ces surenchères correspondaient au moins en partie à une certaine réalité, puisque l'Etat disposait de deux atouts majeurs: d'une part, l'existence d'un stock important de logements vacants; d'autre part, l'appui de l'armée, seule institution disciplinée et efficace. Mais la mise en oeuvre de toutes ces mesures allait se heurter à une machine bureaucratique enracinée de longue date, qui n'allait pas se découvrir une dynamique subite ni sortir de sa léthargie du jour au lendemain. De même que les premières estimations des dégâts ne donnaient pas la mesure réelle de leur importance ( $\mathrm{d}$ 'abord estimée à plus d'un milliard de LE, la facture fut ensuite multipliée par quatre), les dons furent largement inférieurs à toutes les prévisions (200.000 LE). S'ajoute à ceci la faiblesse des effectifs pouvant assumer les tâches requises dans les délais déterminés. En dépit, donc, des bonnes intentions du gouvernement, les mécanismes sociaux et institutionnels allaient entraver les efforts entrepris et mettre en cause la crédibilité du discours officiel. Phénomène qui n'est pas surprenant dans un système où le projet et son exécution, le dire et le faire, le droit et le fait ne concordent que rarement, et où les 
plans les plus élaborés, les projets les plus généreux, sont toujours inopérants, voire pervertis.

L'inadéquation entre moyens et fins n'inciteront pas le gouvernement à donner suite à l'appel pressant lancé par Ibrahim Chukri, chef du Parti socialiste du Travail, destiné à "mobiliser toutes les forces vives de la nation pour atténuer le plus rapidement possible les souffrances humaines » (al-Cha'b,16/10). Le monopole de l'action sociale par le gouvernement, en ce qu'il visait essentiellement à marginaliser les militants islamistes, paraissaient inopportun aux yeux de beaucoup: "Ce n'est pas le moment d'exacerber le conflit avec les islamistes ", estimait Sa'd al-Din Ibrahim, avant d'ajouter qu'il s'agissait là d'un " conflit relevant d'une stratégie étrangère aux impératifs de la crise actuelle. Par ailleurs, nul ne doute de l'incapacité de la machine bureaucratique à mettre en œuvre les initiatives du gouvernement. » (al-Mugtama' al-Madani, novembre 92)

En effet, l'impéritie de l'action gouvernementale ne tardera pas à se manifester. Le débordement des services de quartier, de district et de gouvernorat ne parvenant pas à faire face aux avis d'effondrement et aux demandes d'expertise, ajouté à la lourdeur des démarches administratives, va entraver le déroulement logique des procédures envisagées : «L'établissement d'un procès-verbal pour l'effondrement d'un immeuble ne se traduit pas automatiquement par le relogement et le versement des indemnités promises. » (al-Gumhuriyya, 18/10). Les délais peuvent atteindre plusieurs jours, voire plusieurs semaines. Même pour l'obtention du strict nécessaire, la machine bureaucratique se met en marche, comme en témoigne $M$. Zaynhom, député de Gamaliyya : «Pour obtenir des couvertures ou des vivres, je dois adresser une note au gouvernorat, puis au Ministère dès Affaires sociales, puis au vice-ministre et enfin au gouverneur... Ma note est soumise à examen, et lorsqu'elle est ratifiée, un fonctionnaire vient faire un constat, à l'issue duquel il faut encore attendre sa réponse. Est ce imaginable? »(Sabâh al-Khayr, 29/10)

A la lourdeur bureaucratique s'ajoutent divers abus tels que le détournement de l'aide et des dons, le clientélisme, le favoritisme, mais aussi l'incompétence des ingénieurs qui effectuent les expertises : d'un côté, « certains propriétaires exagèrent les dégâts pour obtenir l'autorisation de démolition....»; de l'autre, "un des candidats du Parti national démocrate, à Gamaliyya, a obtenu sept appartements en contrepartie de son logement détruit par le séisme...» (Sabâh al-Khayr, 29/10). Al-Cha'b dénonce "le gouvernement qui s'empare des dons et laisse les citoyens dans la rue » (23/10) et alWafd titre « 500 familles campent dans la rue à Bab al-Cha'riyya, au moment où les appartements sont distribués aux plus favorisés » (17/10).

De nombreux citoyens ont noté l'ambiguïté des critères de sélection des ayants droit au relogement: "Abd al-Halim, comptable au Ministère de l'Intérieur, qui habitait au sixième étage d'un immeuble à 'Abdin, a vu son plafond s'effondrer et des fissures verticales se former le long des murs; il a fait établir deux procès-verbaux, les 15 et 16 octobre, au qism de 'Abdin, mais c'est son voisin, vivant à l'étage en dessous, qui eu droit à un logement dans la ville d'al-Salam. » (al-Gumhuryyah 18/10)

Mais les infortunes des habitants du Caire apparaissaient dérisoires à côté de la misère des sinistrés des villages de Giza et du Fayoum. Réfugiés dans les champs et le long des berges des canaux, souvent dans des abris de fortune, ce ne sont pas des logements qu'ils revendiquaient mais des tentes ou, tout simplement, la visite d'un responsable. Jusqu'au 17/10, ils n'avaient bénéficié d'aucun secours matériel ni soutien moral: 
« Même pour transporter les blessés dans les hôpitaux, ils ont eu recours au bétail... » (al-Wafd, 18/10)

L'ensemble de ces éléments va créer les conditions objectives d'une explosion. Des manifestations éclatent au Caire et à Giza le 17/10. Deux groupes de manifestants de Sayyida Zaynab se dirigent vers le Parlement par la rue Qasr al-Ayni en lançant des slogans hostiles au gouvernement et au Parti national.

51 Jouant de son charisme et de sa popularité, Fathi Surur, actuel président de l'Assemblée et député de Sayyida, parvient à calmer les manifestants, qui se dispersent au bout de deux heures. A Bulaq en revanche, la situation est plus tendue : pendant 6 heures, les manifestants bloquent la circulation, mettent le feu à un kiosque et lancent des pierres contre le poste de police. Les forces de l'ordre ripostent. Bilan : de nombreux blessés et 62 arrestations (al-Ahâli, 21/10). Commentant ces événements regrettables, le chef du poste de police de Bulaq les a curieusement justifiés en rappelant que «sur les 1.300 avis d'effondrement enregistrés dans le qism, 700 cas ont reçu un bon de relogement, mais parmi eux, seuls 321 ménages ont effectivement été relogés ou hébergés provisoirement. » (al-Ahâli, 21/10)

52 A Giza, quelques manifestants se sont attroupés devant le siège du gouvernorat pour exposer leurs revendications, et une délégation a été reçue par certains responsables pour tenter de contenir la situation. Quelques jours plus tard, le gouverneur de Giza qualifiait l'attitude des manifestants de "manque de civisme » et les accusait d'avoir « fait perdre deux heures aux responsables » (al-Ahâli, 21/10).

Dans ce contexte, la polémique s'est encore exacerbée. Accusé de prévarication par le Parti du rassemblement progressiste, le pouvoir a été complètement discrédité par le Parti du travail, qui a appelé les Egyptiens à « balayer ce gouvernement incapable avec les décombres du séisme, et à faire confiance à ceux qui ont une vocation naturelle à exercer le pouvoir. » (al-Cha'b, 20/10)

De leur côté, les journalistes proches du pouvoir dénonçaient ces attaques, mettant en cause ceux qui sont "incapables d'agir» et "tout juste bons à dénigrer l'action des autorités, à manipuler les pauvres sinistrés en exploitant la crise et en les poussant à la révolte dans un contexte où l'on devrait faire preuve de solidarité... " (S. Muntasir, M. al-Ansari al-Bacha, October, 25/10)

Malgré ces contre-attaques, l'embarras du pouvoir semblait évident. Les responsables ont commencé à s'interroger sur leur manière de gérer la crise :

" Avons-nous failli à nos devoirs? » s'intitulait l'éditorial d'Ibrahim Nâfi', rédacteur en chef du quotidien al-Ahrâm. Et dans deux articles successifs, le président du syndicat des journalistes allait prendre la défense d'un pouvoir auquel il s'était identifié. Commençant d'abord par saluer « le grand peuple qui a révélé sa vraie nature au cours de la crise ", il s'attaquait ensuite à « la minorité des professionnels de la politique, qui essaient de faire commerce des douleurs du peuple par le biais de calculs électoraux minables, au moment où nous nous consacrons jour et nuit au soin des blessés, au relogement des rescapés, et où nous apportons notre soutien moral à ceux qui ont perdu un proche... » Il énumérait pour finir tous les efforts entrepris par l'Etat, " incarné par son chef », et par le gouvernement, représenté par son premier ministre : "Quinze minutes après le tremblement de terre, l'Égypte a mobilisé tous ses moyens pour faire face aux conséquences du désastre. Une commission ad hoc a été mise sur pied au sein du Conseil des ministres, l'état d'urgence a été décrété dans tous les 
hôpitaux, où les médecins étaient appelés à rejoindre leur poste. Les unités de défense civile ont été dépêchées sur les lieux ; en 24 heures, les ambulances ont effectué 13.000 déplacements et transporté 8.000 blessés, tandis qu'on procédait à 9.000 radiographies et que l'on opérait 1.800 fractures... » (...) «Ainsi, fut accomplie la première tâche, à savoir le déblaiement des décombres et le secours aux blessés » (...) « Le relogement des sinistrés était la deuxième tâche d'importance : le gouvernement a réussi à reloger en 24 heures 3.000 citoyens à Madinat al-Salam» (...) «Parlons de la question du relogement : quel pays au monde serait capable de reloger ou d'héberger 7.000 à 8.000 ménages en 24 heures ? Les Etats-Unis ne sont pas encore venus tout à fait à bout des conséquences du cyclone qui a frappé la Floride il y a quelques semaines... Au Mexique et en Argentine, dans des circonstances analogues, on a mis six mois à reloger la population... D'autre part, aux Etats-Unis, le pays le plus riche du monde, l'Etat ne prend pas en charge la reconstruction des logements effondrés, mais se contente de garantir les prêts contractés auprès des banques par les sinistrés. » (al-Ahrâm, 20/10) «Or, en Égypte, l'Etat a promis de prendre en charge même la restauration des édifices endommagés. » (...) «La troisième tâche, qui consistait à réparer les moindres dégâts dans les installations dites "à haut risque » (c'est-à-dire celles pour lesquelles des avaries même mineures peuvent avoir des suites extrêmement graves pour l'environnement et la population, comme les industries chimiques traitant ou stockant des produits hautement toxiques et susceptibles de se répandre, le Haut-Barrage, les puits de pétrole, ainsi que nombre d'infrastructures dont le dysfonctionnement peut provoquer des pertes économiques considérables) et à les remettre en état de fonctionnement, se déroula dans la plus grande discrétion et fut effectuée par des équipes grâce auxquelles il n'y eut ni coupure de courant, ni coupure d'eau, ni interruption des communications, ce qui permit aux citoyens de s'enquérir de la santé de leurs proches après le séisme, tant à l'intérieur qu'à l'extérieur du pays. » (al-Ahrâm, 20/10, 23/10).

57 Ce plaidoyer complaisant, visant à disculper le pouvoir et à redorer son blason, n'a pas empêché les parlementaires de dénoncer certaines lacunes: «Au cours d'une séance animée au parlement, la commission des propositions et des réclamations a attaqué le Ministère des Affaires sociales, le mettant surtout en cause pour n'avoir pas incité les associations qui lui sont affiliées à jouer un rôle dans la crise en accueillant et assistant les sinistrés, à l'inverse des syndicats qui se sont montré plus actifs. Par ailleurs, la commission a déploré l'absence des responsables du ministère sur les lieux les plus affectés, en particulier les villages de Giza et du Fayoum... Les députés ont ensuite relevé les contradictions des bilans fournis par le gouvernement. » (al-Akhbâr, 21/10\}

Loin des querelles politiciennes et des positions partisanes, le gouverneur du Caire relate, dans une interview accordée à l'hebdomadaire al-Musawwar, la chronologie des faits, en mettant en lumière les tâtonnements des débuts, les limites des moyens d'intervention et les tentatives de maîtrise de la crise. Nous citons ici de longs extraits de ses déclarations, particulièrement claires et impartiales.

«Au début, ce fut la panique générale. Nous nous sommes trouvés submergés par un déluge d'avis d'effondrements ou de fissures et des demandes d'expertises - 50.700 avis pour le seul gouvernorat du Caire jusqu'au 20/10. Conscients du manque d'effectifs - le nombre des commissions d'expertises mises sur pied étant largement insuffisant pour faire face à la demande - nous avons demandé des renforts : 240 ingénieurs ont été mis à là disposition du gouvernorat par l'armée (160) et par le Ministère de l'Habitat 
(80). Entre le 20/10 et le 6/11,42.100 édifices ont été expertisés, sur les 50.700 répertoriés jusqu'au 20/10. Il en restait donc 7.469, auxquels il faut ajouter ceux qui étaient en attente depuis le 20/10, outre les immeubles déclarés hors d'usage avant le séisme (estimés à 3.500 par an). Tout s'accumulait et interférait... Le deuxième problème auquel il fallait faire face, et qui augmenta notre désarroi, était l'augmentation continue et imprévisible des sans-abri ; en effet, les constats d'expertise avaient fait état de dégâts importants dans les vieux immeubles, ce qui nécessitait une évacuation immédiate des habitants. Ceux-ci se réfugiaient dans les rues car nous n'avions pas prévu des centres d'hébergement provisoires pour tant de citoyens. C'est à ce moment que les islamistes sont intervenus dans les quartiers populaires, y dressant des tentes portant leur slogan (« l'Islam est la solution »), mais sans nous avertir et sans tenter de coordonner leur action avec la nôtre. Ces pratiques à visée purement électorale ont provoqué la colère des représentants locaux du Parti national au pouvoir, qui nous ont demandé d'intervenir pour mettre fin à cette exploitation intentionnelle de la crise. (...) Par ailleurs, j'ai refusé de donner suite à la demande émise par le syndicat des ingénieurs (largement noyauté par les islamistes) de collaborer avec nous, dans la mesure où je disposais d'un effectif suffisant d'universitaires très compétents qui nous servaient de consultants... " (al-Musawwar, 11/11)

En ce qui concerne le relogement et l'hébergement des sinistrés, la situation se présentait comme suit au 6 novembre ;

61 - 3.945 ménages logés à Madinat al-Salam et à Diwiqa ;

62 - 3.115 ménages hébergés dans les camps aménagés par les forces armées à Madinat alSalam, à Helwan et au Haikstep ;

$63-1.817$ ménages hébergés dans 20 centres de jeunesse ;

64 - 6.758 ménages hébergés chez leurs proches. Les besoins initiaux en unités de relogement s'élevaient à 15.635, mais les besoins réels furent évalués, après expertise, à 26.221 (voir tableau ci-dessous). Compte tenu du nombre des ménages logés, hébergés dans des camps ou chez des proches, il restait donc 10.528 ménages dans la rue ou dans des immeubles qui menaçaient de s'écrouler à tout moment.

\begin{tabular}{|l||l|l|}
\hline Type de dégâts & Nombre d'immeubles & Nombre d'unités de logement \\
\hline \hline Immeubles totalement effondrés & 102 & 585 \\
\hline \hline Immeubles hors d'usage, à démolir & 3.146 & 11.440 \\
\hline \hline & & 24 chambres \\
\hline \hline Immeubles devant subir des & & \\
\hline \hline démolitions partielles & 7.384 & 14.117 \\
\hline \hline & & 55 chambres \\
\hline \hline Immeubles à restaurer & 19.854 & \\
\hline
\end{tabular}




\begin{tabular}{|l|l|l|}
\hline Immeubles demeurés intacts & 9.061 & \\
\hline \hline Total des unités de logement requises & 26.142 \\
\hline \hline & +79 chambres \\
\hline
\end{tabular}
manœuvre politique destinée à améliorer l'image de marque du pouvoir à l'étranger.. Quant aux citoyens ayant indûment bénéficié d'une unité de relogement, ils se sont manifestés d'eux-mêmes à la suite de la promulgation d'un décret présidentiel châtiant de 15 ans de travaux forcés les coupables de telles pratiques (al-Ahrâm, 21/10) : 225 personnes ont ainsi rendu leur clef au gouvernorat du Caire. Le président de la République avait révélé, dans une conférence de presse tenue le 18/10, l'existence de certains abus : "Sur 1.516 sinistrés hébergés provisoirement dans le camp d'al-Khanka, auxquels le président a rendu visite, 516 seulement pouvaient prétendre à une unité de relogement. Les 1000 autres ont essayé d'exploiter la situation en fournissant de fausses données. » (al-Ahrâm, 18/10)

La critique de l'Etat providence

69 Si l'essentiel de la critique formulée à rencontre du pouvoir concernait les défaillances de la gestion de la crise et, paradoxalement, son monopole, certaines critiques se sont cependant axées sur l'excès de l'interventionnisme étatique et la passivité de 
l'ensemble de la société civile. Mais cette dernière, remarque le philosophe Hasan Hanafi, « est écartée de toute participation depuis 40 ans et s'est donc habituée à ce que l'Etat fasse tout à sa place, ce qui a eu pour conséquences d'affaiblir ses capacités d'initiative. »(al-Mugtama' al-madani, nov. 92). Dans le même esprit. M. al-Husayni souligne les limites de l'initiative privée dans une société qui s'est engagée sur la voie du libéralisme économique et politique mais où chacun veut bénéficier des avantages sans en assumer les contraintes: "Lorsqu'une société affronte un grave cataclysme, il est normal qu'elle mobilise toute son énergie pour y faire face. Or, à la suite du tremblement de terre qui a frappé récemment notre pays, on constate qu'on est loin de cette mobilisation. N'apparaissent que les efforts de l'Etat et les dons versés par quelques organismes, institutions ou individus. Mais force est d'avouer, même si cela semble choquant pour certains, que ces dons demeurent largement en deçà du nécessaire... Cessons donc le flot de louanges sur la solidarité... Une altitude solidaire ne devrait pas être un geste de charité ou de prodigalité laissé à l'initiative de chacun, mais une charge sociale obligatoire, précise et organisée... .(..) La responsabilité de l'Etat dans ce genre de situation devrait se limiter aux secours aux sinistrés, dans un premier temps; la société civile devrait ensuite prendre le relai, le rôle de l'Etat étant alors d'organiser la prise en charge sociale.... Quelques exemples : les écoles récemment construites et qui se sont effondrées au cours du séisme devraient être reconstruites au frais des entrepreneurs, qui devraient également verser des indemnités aux familles des victimes... La restauration des monuments historiques devrait être financée en grande partie par les entreprises de tourisme qui, grâce à ce patrimoine, ont accumulé des fortunes... » (al-Musawwar, 30/10)

A ce discours rationnel qui constitue une réflexion de fond sur les problèmes agitant la société égyptienne d'aujourd'hui, s'opposait un discours religieux culpabilisant et moralisateur.

Islam et séisme

71 Calamité naturelle, le séisme se prêtait plus que tout autre phénomène social ou économique à une interprétation religieuse. «Châtiment » et « repentir » en furent les mots clés. Quelques nuances furent cependant introduites pour définir la charge symbolique du séisme : avertissement, mise à l'épreuve, colère divine ou tout cela à la fois, comme l'exprimait 'Adil Husayn dans un article publié par al-Cha'b: "Les catastrophes naturelles constituent une mise à l'épreuve pour la nation et les organes de l'Etat... Nous y avons été exposés à plusieurs reprises ces derniers mois, à Edkou, à Beheira, lors du naufrage du Salem Express. Mais le tremblement de terre diffère, par son ampleur et sa signification, de tous les désastres qui l'ont précédé. Comme les éruptions volcaniques, les crues, les cyclones et les tempêtes, le séisme rappelle à l'individu conscient le vrai poids de l'être humain, il lui rappelle la supériorité du TrèsHaut, qui commande et sait tout...»

Citant ensuite des versets du Coran à l'appui de ses propos, l'auteur énumérait les péchés commis par la société ayant mérité ce «châtiment»: profits illicites, consommation d'alcool, débauche, "et à un autre niveau, plus politique que moral: l'abandon de l'Irak, de la Somalie, de la Bosnie-Herzégovine, de Jérusalem... » (al-Cha'b, 16/10). Le penseur islamiste M. al-Chakaa partageait ce point de vue: «Certes, le séisme est un châtiment de Dieu, mais dans le cas de l'Égypte, j'y verrais plutôt un avertissement, étant donné la faible puissance du séisme. » (al-Nûr, 21/1,0). Le cheikh Cha'rawi va plus loin : "C'est une répétition du jour du jugement dernier, estime-t-il, 
un examen de la foi et de la soumission des vrais croyants, » (as-Siyâsi al-Masri, 25/10) AL-Hamza Deibess rejette quant à lui l'idée d'un châtiment collectif au profit de celle du châtiment individuel : une seule personne, le ministre de l'Education Husayn Kamal Baha al-Din, aurait provoqué la colère divine : " A cause du blâme qu'il a adressé à la directrice de l'école Qâsim Amin, qui imposait à ses élèves le port du voile, la réponse du ciel n'a pas tardé : 550 écoles se sont effondrées. » (al-Nûr, 21/10) Le cheikh Kichk soutient lui aussi cette thèse puisqu'il voit dans le séisme «le châtiment des mécréants et une épreuve pour les croyants, les premiers devant se soumettre à l'attrition, les autres au repentir» (al-Nûr, 21/10). Châtiment, avertissement ou signal d'alarme, «Dieu a pourtant été miséricordieux, car si la secousse avait été plus forte d'un degré, Le Caire se serait entièrement effondré. » (M. al-Hayawan, al-Gumhuriyya, 14/10) « C'est grâce à tous les musulmans qui étaient prosternés dans les mosquées au moment du séisme que Dieu a été clément ", soutient à son tour le cheikh Isma'il al-Adawi dans son sermon du vendredi à al-Azhar (al-Liwa al-lslami, 22/10). La "miséricorde " et la " générosité » de Dieu vis-à-vis de l'Égypte se manifestent par la faiblesse des pertes humaines et matérielles subies: "Qu'un tremblement de terre aussi puissant ne provoque pas plus de ravages, que le nombre des victimes soit moins important que dans le cas d'un accident de la route, ce sont là les signes de la protection divine de l'Égypte. Ce pays restera à jamais protégé car Dieu l'a recommandé à son Prophète... » (F. Hachem, al-Ahrâm, 14/10) Mais c'est « toute la nation islamique que Dieu a favorisée et élue parmi toutes celles qui l'ont précédée ", (al-Liwa' al-islâmi, 15/10). Par conséquent, le séisme apparaît moins comme un châtiment ou un avertissement que comme une mise à l'épreuve pour tester la foi des croyants qui « ont fait preuve d'une grande solidarité et d'un franc dévouement » (ibid). Et " comme toute récompense doit être méritée », selon le Dr. A. Chahin, «il faut affronter des épreuves difficiles sur terre pour mériter le paradis dans l'au-delà » (Ibid).

Il n'est pas rare de trouver également des interprétations tout à fait rationnelles malgré le recours au réfèrent religieux. C'est le cas chez le cheikh Attiya Saqr qui, refusant l'idée de châtiment collectif mérité par la non application de la charî'a, estime que les Egyptiens suivent la charî'a plus que partout ailleurs dans le monde : "Ils remplissent les mosquées, effectuent le pèlerinage à la Mecque, observent le jeûne au mois de Ramadan... Rappelons que l'Égypte n'est pas le seul pays musulman à avoir été frappé d'un tel désastre, et ce n'est pas un phénomène sans précédent dans l'histoire du monde musulman... (...) Peste et disette se sont abattues sur les musulmans à l'époque de 'Umar, et à aucun moment il n'a été question de châtiment... Or, à l'époque, les musulmans étaient encore plus conscients de l'essence véritable de l'Islam et de ses objectifs. » (al-Siyâsi al-misri, 18/10) Ce point de vue éclairé est partagé par M. alFayoumi, doyen de la Faculté des études islamistes (Ibid).

Ces avis raisonnés n'ont pas empêché que se développe dans la presse une campagne de dénonciation et de culpabilisation amplifiée par les milliers de hauts-parieurs des mosquées qui quadrillent tout le territoire. Cette campagne visait tant les simples citoyens, dont les " cœurs se sont endurcis ", que les mécréants qui «consomment de l'alcool et produisent des films indécents ", « les communistes et les athées tolérés par le pouvoir ", « les femmes qui ne portent pas le voile », " les corrompus, les hypocrites, les despotes » (al-Gumhuriyya, 14/10, al-Akhbâr, 29/10) et, bien évidemment, l'Etat luimême dont l'incompétence a éclaté au grand jour grâce au séisme : "Dieu a voulu que le séisme ait lieu au moment de la campagne pour les élections des conseils locaux, afin 
de dévoiler la corruption qui s'est généralisée dans notre société au cours de ces dernières années et afin de mettre en évidence l'incapacité de l'Etat. » (al-Cha'b, 16/10)

Les témoignages de repentir se sont multipliés : «Le premier pas vers le repentir, c'est le retour à ma femme »; «Je ne vais plus tricher aux examens »; "J'ai décidé de porter le higâb pour me rapprocher de Dieu, car notre immeuble est tellement fragile qu'il risque de s'écrouler à tout moment »; «Je regrette d'avoir passé ma vie à accumuler de l'argent » (October,17/10).

76 Le tremblement de terre appelle la rédemption. 'Adil Husayn prie "pour que le repentir des 20 millions des citadins qui ont vécu le séisme soit authentique, que l'avertissement puisse transformer les âmes » (al-Cha'b, 16/10).

La fragilité des constructions

77 "Croit-on que la catastrophe va nous changer? Un peuple ne peut changer du jour au lendemain.» (Sabâh al-Khayr, 26/10) En effet, si les catastrophes transformaient les peuples, cela aurait dû se produire, depuis dix ans que s'effondrent les immeubles anciens ou récents, provoquant autant de dégâts matériels et de pertes humaines, sinon davantage, que le séisme du 12 octobre...

Selon l'Encyclopedia Universalis, "Les effets d'un tremblement de terre sur les constructions humaines dépendent essentiellement, à intensité égale, de la qualité des édifices. Si rien ne semble pouvoir résister à une intensité maximale (XII sur l'échelle de Mercalli), les zones limitées par les isosestes XI, X, et même IX peuvent être entièrement ravagées si les bâtiments sont mal conçus, ce qui arrive dans la plupart des pays dits développés, ou bien, lorsque les constructions sont de type parasismique, ne présenter que des dégâts relativement réduits, et en tout cas moins meurtriers. Pour résister à une secousse, un édifice doit posséder une certaine élasticité et une certaine cohésion: les maçonneries hétérogènes, celles dont le mortier est médiocre, les bâtiments dont le centre de gravité est placé trop haut, les toitures trop lourdes, les vérandas, les encorbellements, tout cela peut s'effondrer, parfois dès l'intensité VIII. C'est ainsi que, dans la même année 1960, un choc superficiel d'une amplitude inférieure à 6 , celui d'Agadir, a tué autant de personnes que le colossal tremblement de terre du Chili, d'une amplitude de 8,6 à 8,7. Quoique de qualité assez médiocre, les bâtiments de Valdivia où de Conception étaient néanmoins mieux construits que ceux d'Agadir. De plus, alors que les gourbis de mauvaise et lourde maçonnerie s'écroulaient au Maroc, les maisons de bois qui constituent l'essentiel des bourgades et des villages du sud du Chili résistaient fort bien aux secousses grâce à leur élasticité. » (3)

Ces éléments d'analyse des effets des tremblements de terre sur les constructions viennent à l'appui de l'idée, partagée par de nombreux spécialistes égyptiens, selon laquelle le tremblement de terre du 12 octobre, d'une amplitude inférieure à $6, \mathrm{n}$ 'est pas entièrement responsable des dommages causés aux constructions en milieux urbain et rural. C'est l'état de délabrement avancé des vieux immeubles et la mauvaise qualité des constructions neuves qui sont à mettre en cause. Certains s'attendaient même à des dégâts plus importants : " On a tort de croire que le séisme du 12/10 est un séisme destructeur. $\mathrm{N}$ 'exagérons rien : les dégâts sont insignifiants dans un pays qui ne soucie guère de la qualité des constructions." (al-Ahrâm, 17/10) Ce constat, qui contraste avec l'avis général sur l'importance des destructions, se base sur des données vérifiées. En effet, le nombre total des bâtiments, toutes catégories confondues, qui se sont écroulés ou ont été endommagés ne représente que $0,25 \%$ du total des bâtiments en Égypte (19.248, sur 7.706.330 bâtiments\}. Mais le problème est «moins celui des 
édifices qui se sont effondrés que celui de ceux qui vont s'écrouler » (al-Cha'b, 16/10). $C^{\prime}$ est sur cet aspect de la crise du logement en Égypte que la presse a mis l'accent, avec des révélations qui donnent le vertige... Ainsi peut-on lire dans al-Wafd: « Attention, à la prochaine catastrophe, 20 millions d'Egyptiens risquent de se retrouver sans abri : il y a 2.215.000 logements vétustés en Égypte... Ces chiffres effrayants, nous ne les avons pas inventés : ils sont issus d'un rapport élaboré par une commission spécialisée, instance gouvernementale. Malheureusement, ce rapport n'a jamais retenu l'attention des responsables. Parmi ces logements figurent les HLM construits à l'époque nassérienne, jamais entretenus et qui, de surcroît, ont subi de multiples ajouts... Le gouvernement, à la lumière de ces données, s'est contenté de prendre des décisions d'évacuation, mais les habitants, à leur risques et périls, ont refusé d'évacuer ces immeubles, allant même jusqu'à se pourvoir en cassation contre les décisions de démolition (300.000 pourvois pour la seule ville du Caire), préférant mourir sous les décombres. Car l'alternative était la rue, ou au mieux, pour les heureux élus, les habitations d'urgence du gouvernorat. » (al-Wafd, 15/10)

Deux autres journaux, al-Cha'b et al-Ahrâm at-Masâ'i, ont confirmé les données du rapport de la commission spécialisée cité par al-Wafd. Aucun démenti des membres de la dite commission n'est venu contredire ces informations. De plus, le grand spécialiste égyptien de la question du logement, Milad Hanna, a estimé le pourcentage des logements vétustés au Caire à $25 \%$ du parc, auxquels il faut ajouter $40 \%$ des vieilles habitations qui menacent de s'écrouler (al-Gumhuriyya, 15/10). Citant un rapport technique produit par la direction du logement du gouvernorat du Caire, l'hebdomadaire al-Musawwar parle de $60 \%$ d'édifices vétustés au Caire, et de $70 \%$ de bâtiments construits sans permis et sans architectes (al-Musawwar, 23/10). De son côté, l'Université d'al-Azhar a effectué une étude portant sur 11 secteurs du Caire, étude dont les résultats, exposés au cours d'un colloque tenu en Égypte en 1990, montrait que $60 \%$ des logements dans les secteurs étudiés menaçaient de s'écrouler (al-Musavwar, 23/10). Ces chiffres qui tantôt se recoupent, tantôt se contredisent, s'ils sont quelquefois sujets à caution, n'en révèlent pas moins l'état déplorable du bâti en Égypte, et en particulier au Caire.

81 Les causes de cette situation sont bien connues. Dans la vieille ville, c'est l'absence d'entretien, l'augmentation des densités et, plus récemment, la remontée de la nappe phréatique et des eaux usées qui ont contribué à la détérioration et à la ruine des habitations dans ces secteurs. Al-Ahrâm al-Masấi dresse l'état des constructions de la vieille ville avant le séisme : «A Gamaliyya, Bâb al-Cha'riyya et Darb al-Ahmar, 352 cas d'évacuation ont été décidés en trois mois." Citant un rapport technique du gouvernorat, le quotidien atteste que « 4 édifices étaient déclarés hors d'usage tous les jours au Caire ». Par ailleurs, des milliers de décisions d'évacuation et de démolition pour cause de vétusté ne furent pas exécutées : 3.500 entre 1986 et 1988, 2.000 en 1991..Elles ont atteint 16.000 en 1992 ! (al-Ahrâm al-Masâ'i, 12/10). Si, donc, des milliers de décisions de démolitions avaient été adoptées avant le 12 octobre, le séisme n'a fait qu'accélérer un processus qui était en cours, ou plutôt, il a exécuté des décisions qui étaient restées lettre morte à ce jour. On peut donc "remercier le séisme », à l'instar d'une journaliste de Sabâh al-Khayr (26/11 ), non seulement parce qu'il a «fait le travail » mais aussi parce que les rescapés bénéficieront de logements neufs, étant donné le caractère exceptionnel de la situation. Seulement, le séisme n'a accompli qu'une infime partie du travail: des dizaines de milliers d'immeubles vétustes sont encore debout, mais ils sont sérieusement endommagés, à tel point que «le passage 
d'un tracteur dans la rue peut provoquer leur écroulement ", comme le constate avec humour le président du département du béton armé au Ministère de l'Habitat (Huruyyati, 18/10).

82 Les débats sur la fragilité de l'ancien parc locatif n'ont pas soulevé les questions de rénovation, de restauration et de réhabilitation urbaine. Certes, on va restaurer les bâtiments fissurés, mais l'instauration d'un système capable d'assurer l'entretien systématique du patrimoine, au sens le plus large, n'est toujours pas à l'ordre du jour. L'effondrement des vieux immeubles apparaît comme une fatalité car, selon une idée très répandue, « ces immeubles ont atteint l'âge limite ». «Si tel était le cas, il faudrait démolir les Pyramides... Un édifice n'a pas de limite d'âge. Il peut durer des siècles s'il est bien construit au départ et bien entretenu par la suite ", rétorque Milad Hanna avant d'ajouter que «les vieux immeubles constituent un patrimoine mondial qu'il importe d'entretenir systématiquement. On ne doit avoir recours aux démolitions et aux déplacements de population que dans des cas extrêmes. » (al-Ahâli, 28/10)

Le syndrome des effondrements de constructions neuves

83 "Lorsque le séisme s'est produit, j'ai tout d'abord cru que la tour voisine allait s'effondrer sur notre immeuble... On en a tellement l'habitude depuis un certain temps!» (propos du gouverneur du Caire, al-Musawwar, 6/10) Le gouverneur du Caire n'a pas été le seul à redouter un effondrement dès la première secousse. C'est une crainte que partagent la plupart des Egyptiens, qu'ils occupent « des édifices construits par le secteur public ou par le secteur privé» ou qu'ils logent «dans de vieux immeubles ou dans les tours grand standing de l'époque de l'infitâh", comme le souligne le sociologue 'Ali Fahmi avant d'ajouter que « ce syndrome collectif mérite une attention particulière, car il révèle un manque de confiance total dans la qualité des constructions » (al-Qâhira, novembre 1992). Il révèle aussi, estimons-nous, un sentiment d'insécurité générale qui tourmente la population urbaine et qui se justifie par des effondrements à répétition au cours de ces dix dernières années. Une journaliste de Sabâh al-Khayr en a dressé la chronologie :

84 - Mai 1982 : 10 piliers en béton armé éclatent dans le plus grand immeuble de Mohandessin, qui était en cours d'achèvement ;

$85-1984$ : un immeuble s'effondre à Héliopolis, tuant 17 personnes ;

$86-1985$ : le gigantesque immeuble du Maryland, toujours à Héliopolis, s'écroule avant d'être habité ; au cours de la même année, 9 piliers se fissurent dans un immeuble de 9 étages à Doqqi ;

$87-1986$ : un immeuble en cours d'achèvement menaçant de s'écrouler dans le quartier des Pyramides, six édifices situés dans son voisinage immédiat sont évacués ; au cours de la même année, des fissures importantes sont découvertes dans quelque 1.000 unités de logements et 127 bâtiments publics à Port-Saïd ;

$88-1987$ : de nouveau dans le quartier des Pyramides, un immeuble construit depuis six ans menace de s'écrouler : même scénario ;

89 - à Alexandrie, en décembre 1989, les corps de 12 personnes sont exhumés des décombres d'un immeuble à Glim; en 1991, le drame se reproduit à Sidi-Gaber et plus récemment, en 1992, 20 personnes trouvent la mort suite à l'écroulement d'une tour dans le quartier de Loran ;

$90-1991$ : à Isma'iliyya, 53 petits immeubles collectifs s'écroulent partiellement, 7 ans après leur construction... 
91 Cette chronologie demeurerait incomplète si l'on n'y ajoutait les quelque 50 effondrements répertoriés dans diverses villes égyptiennes et ayant fait plus de 100 morts, sans compter les blessés. (Sabâh al-Khayr, 26/11)

Cette série a été couronnée par l'effondrement, provoqué par le séisme, de l'immeuble d'Héliopolis. "Quoi de neuf?» se demande la journaliste, indignée par le débat sans précédent qui s'est développé dans la presse sur le secteur du bâtiment. "Nous connaissons bien les termes de ce débat, qui a défrayé la chronique à plusieurs reprises au cours de cette dernière décennie. Le dossier était ouvert, puis refermé dès qu'étaient désignés les auteurs du crime et dénoncés l'inconscience, les abus de pouvoir, la complicité des services du logement et des permis de construire, la non-conformité des matériaux de construction... » (Sabâh al-Khayr, 26/11)

En effet, le débat sur cette question récurrente paraît stérile dans la mesure où les éléments du dossier sont connus de tous, des spécialistes aux responsables en passant par l'homme de la rue. Les pratiques des petits entrepreneurs du secteur informel font actuellement recette dans l'ensemble du secteur du bâtiment : moins de ciment, moins de fer à béton, moins de mortier, plus de sable, plus d'étages. Minimiser les coûts et maximiser les profits, tel est le nouveau « code de la construction » élaboré et appliqué par les maîtres d'oeuvre en Égypte. Le résultat de ces pratiques défigure nos villes : partout se dressent des bâtisses sans caractère, laides, de mauvaise facture, qui se dégradent avant même d'être habitées ; le travail mal fini, bâclé ou improvisé apparaît d'une façon flagrante le long des murs, flanqués des tuyaux de plomb apparents des installations sanitaires; les taches d'humidité résultant de fuites d'eau marquent la plupart des nouvelles constructions, tant celles dites « de qualité » que les autres. C'est là une des causes principales du vieillissement prématuré des bâtiments. A l'inconscience des entrepreneurs s'ajoute l'incompétence des ingénieurs, « qui ont peu d'expérience et abaissent les coûts pour obtenir le marché » (al-Ahrâm al-Masâ'i, 18/10).

Mais les promoteurs et les clients, y compris l'Etat, sont également responsables de la dégradation du bâti, puisque c'est au moins-disant que l'on confie la réalisation des ouvrages. Lorsque les coûts les plus bas deviennent le seul critère de sélection des offres, la qualité des constructions en souffre forcément. "Cette pratique ", écrit un spécialiste dans al-Wafd, "élimine les maîtres d'œuvre compétents et intègres au profit d'imposteurs souvent en faillite qui ont systématiquement recours à des méthodes illicites pour obtenir des marchés : subornation des comités de sélection, altération des matériaux de construction, violation des règlements figurant dans les permis de construire, etc. C'est comme cela qu'on a tué nos enfants. » (al-Wafd, 18/10)

L'effondrement total ou partiel de 3.050 écoles (soit $20 \%$ des établissements scolaires), dont certaines construites il y a moins de six ans (al-Musawwar, 30/10) a non seulement suscité une vive émotion, mais aussi une remise en question globale, touchant tant la qualité de l'enseignement que celle des établissements et des enseignants. Ces derniers, en effet, ont souvent fait preuve d'une grave irresponsabilité, prenant la fuite lors du séisme et abandonnant à leur sort les écoliers paniqués, dont les bousculades ont provoqué plus de victimes que la chute d'un pan de mur ou d'un bloc de béton (Sabâh al-Khayr, 22/10 ; al-Gumhuriyya, 14/10 ; Rose al-Youssef, 19/10 ; al-Ahrâm al-Masâ'i, 16/10). Une catastrophe plus grave a pu être évitée grâce à la rénovation de 7.500 établissements avant le début de l'année scolaire. 5.000 établissements supplémentaires devraient être construits afin de diminuer les densités actuelles (70 à 90 élèves par classe en province). La mise en œuvre de ce programme ambitieux a commencé avec la 
nomination de M. Husayn Baha' al-Din au poste de ministre de l'Education au début de l'année. Reste à savoir qui garantira la mise en vigueur des règlements de construction. Le code de construction

96 «Depuis la fin des années 50, personne ne respecte plus les règlements de construction ni n'obéit aux contrôles de qualité ; par conséquent, les organismes compétents ont disparu ", confiait à al-Musawwar (23/10) l'ancien président de l'Université de 'Ayn Chams et président actuel des comités d'expertise des établissements scolaires et universitaires. Mettre en vigueur les règlements et faire appliquer les codes de construction promulgués respectivement en 1969 et 1989 est devenu une revendication nationale après le séisme. Les débats sur ces questions ont abouti à la promulgation d'un code de construction parasismique. Ce code a pour objet de définir des zones en fonction de leur sensibilité sismique; dans chaque zone, la mise en œuvre des constructions devra tenir compte du niveau d'intensité probable des secousses. L'entrée en vigueur de ce code, prévue en principe dès janvier 1993, semble d'ores et déjà incertaine. $\mathrm{D}$ 'abord, le respect des normes parasismiques augmenterait les coûts d'au moins $30 \%$, ce qui dépasse largement les moyens de ce secteur. Les niveaux de sécurité et de fiabilité exigés vis-à-vis de risques d'une autre nature (charges d'exploitation, vent, incendies...) sont déjà rarement respectés par les maîtres d'oeuvre. Entre les exigences de sécurité et les coûts des mesures de protection, dans un pays où la probabilité d'occurrence d'un séisme de forte intensité demeure faible, on optera toujours pour le deuxième terme de l'alternative. L'application des règles strictes de parasismicité se limitera aux installations à hauts risques.

Soulignons ensuite que le secteur du bâtiment échappe complètement au contrôle de l'Etat: 80 \% des logements construits en Égypte au cours des 15 dernières années l'ont été en violation de toutes les normes constructives, et le reste n'est pas soumis à un examen drastique : « La loi fixe la hauteur d'un immeuble à une fois et quart la largeur de la rue, mais il suffit de se promener pour en constater la violation. » (al-Gumhuriyya, 20/10) Dans une interview accordée à al-Musawwar, le ministre de l'Habitat reconnaît l'impuissance du gouvernement face aux transgressions systématiques et flagrantes des règlements : "En voyant des immeubles de trente étages bordant des rues de huit mètres, j'ai transmis des plaintes portant ma propre signature aux autorités locales, mais en vain... » (al-Musawwar, 22/10)

98 Le quotidien al-Wafd nous donne la mesure de l'ampleur de ce phénomène : « 36.000 procès-verbaux sont établis chaque année pour la seule ville du Caire contre des promoteurs ayant dépassé les hauteurs figurant sur les permis de construire ou surélevé illégalement des immeubles existants. Par ailleurs, 1.500 tours récemment construites à Maadi, Giza, Zamalek et Madinat Nasr ont obtenu des dérogations leur permettant de dépasser les hauteurs autorisées. » (al-Wafd, 22/10) Ces pratiques illicites ont « coûté à l'Etat 5 millions LE afin d'augmenter la capacité des réseaux divers pour la seule année $1991 »$ (al-Gumhuriyya, 20/10).

On se trouve dans une situation où non seulement on laisse faire sans aucune sanction mais on bénit, on récompense même les promoteurs immobiliers en valorisant leurs investissements illicites. Certains expliquent le laxisme des autorités locales vis-à-vis de ces pratiques par l'indifférence, voire la corruption des ingénieurs chargés de l'octroi des permis de construire et de la surveillance des chantiers. La faiblesse du traitement mensuel de ces fonctionnaires entame leur probité et lamine leur résistance à la subornation: de censeurs, ils deviennent complices. D'autres estiment que le manque 
d'effectifs et de moyens ne permet pas, de repérer tous les cas illégaux ou de surveiller étroitement les chantiers pour contraindre les entrepreneurs à respecter le cahier des charges et à raser systématiquement les étages illicites. Faute de pouvoir mettre en vigueur les règlements, les autorités locales ont souvent recours à des accords à l'amiable ; le promoteur paie une amende insignifiante (10 à 30 LE) pour chaque étage construit illégalement et échappe ainsi à toute sanction. Cette procédure évite aux autorités l'hostilité des acquéreurs aussi bien que celle des entrepreneurs-promoteurs auxquels leur puissance financière et politique a permis de faire «main basse-sur la ville » depuis deux décennies.

Le lobby des entrepreneurs

100 Relatant la chronologie des transformations subies par le secteur de la promotion immobilière, Milad Hanna retrace les étapes de la naissance et de la consolidation du pouvoir des promoteurs: "Avec le début de l'infiîah en 1974, l'association des ingénieurs a rassemblé des organismes américains autour d'une table ronde portant sur les règlements devant régir la construction des tours. Ce fut le point de départ de l'introduction de ce type de constructions en Égypte. Certains bureaux de consultants locaux s'y lancèrent, dans un contexte où les prix des terrains sur les berges du Nil et le long de la corniche d'Alexandrie avaient flambé. Une dérogation aux règlements existants était nécessaire.

Le ministre de l'Habitat n'a pas hésité, a même encouragé ces initiatives. Ainsi ont vu le jour en Égypte de nombreuses sociétés immobilières qui se sont spécialisées dans la construction de tours incluant bureaux, commerces, habitat et loisirs. Entre 1980 et 1992, les prix des appartements ont quintuplé, des fortunes considérables se sont ainsi constituées en peu de temps. Des réseaux de relations complexes se sont établis entre les promoteurs entrepreneurs d'une part, les organes des conseils locaux, les notables gouvernementaux tels que les gouverneurs, les anciens et actuels ministres de l'autre. Les promoteurs immobiliers sont ainsi devenus le principal groupe de pression, dont la puissance dépasse celle des associations d'hommes d'affaires... Ces groupes incluent des juristes de renom - l'un d'eux était professeur à l'université et occupa le poste de ministre de la Justice à une certaine époque -, des comptables non moins compétents leur servent aussi de consultants : l'un d'eux est devenu le président d'une commission parlementaire...»

102 Poursuivant son analyse, Milad Hanna explique comment procèdent les promoteurs pour contourner les règlements : «On prépare deux dossiers pour obtenir le permis de construire : celui qui est présenté aux autorités est conforme aux règlements, l'autre contient les vrais plans d'exécution... Lorsque l'édifice est achevé, les autorités constatent avec surprise qu'il possède dix à quinze étages de plus que prévu! » (al-Wafd, 22/10)

103 Tandis que le ministre de l'Habitat, M. al-Kafrawi, rend hommage aux entrepreneurs « je suis content qu'existe une Union d'entrepreneurs que je respecte » (al-Musawwar, 22/10), le président de l'Union s'inquiète du nombre d'entrepreneurs non qualifiés : « Parmi les 30.000 entrepreneurs enregistrés à la Chambre de commerce, $50 \%$ sont des croque-morts incompétents. Ils ont réalisé de nombreux édifices dans lesquels sont apparus de graves défauts techniques. » [Rose at-Youssef, 19/10),

104 L'itinéraire de la hajja Kamila, propriétaire de l'immeuble sinistré d'Héliopolis et promoteur immobilier, montre les étapes de l'ascension de la nouvelle couche sociale " qui nous dirige », comme l'avait prédit Sonallah Ibrahim dans son roman Etoile d'Août, 
écrit en 1974 : « Une jeep décapotable, d'une longueur surprenante, nous dépassa, avec un gros type en galabieh au volant et, à ses côtés, une femme tout aussi corpulente, en robe de paysanne, les bras couverts de bracelets en or. Saïd m'apprit que c'était l'intermédiaire qui pourvoyait en main-d'œuvre les chantiers du barrage : il traitait quotidiennement des milliers de contrats saisonniers, sur lesquels il touchait cinq piastres par jour et par ouvrier... » (p. 96) « et vive nos futurs dirigeants » (p. 184).

Chronologie d'une " réussite "

Née d'une famille nombreuse - six frères et sœurs - habitant dans une seule pièce à Bassatine, non loin des cimetières, où le père exerçait le métier de poseur de dalles de caveaux, Kamila perd sa mère à cinq ans. A seize ans, elle épouse Tawfiq, le fils d'un ami de son père et quitte le logement excentré et pauvre de Bassatine pour aller partager avec sa belle-mère un logement tout aussi pauvre, dans la banlieue est de Matariyya. Petit entrepreneur exerçant son activité dans les quartiers informels de la ville, le mari de Kamila réussit à acquérir un terrain à Hilmiyyâ dans les années 50 (le m2 valait alors dans les $3 \mathrm{LE}$ ) et y fait construire un immeuble pièce par pièce, étage par étage. Associé à son frère dans un petit commerce, il parvient, en 1965, à faire construire quatre étages, auxquels il en ajoute deux autres quelques années plus tard. Au début des années 70 , grâce à la vente du ciment au marché noir, le niveau de vie de cette famille augmente de façon spectaculaire.

Tawfiq échange sa moto contre une Fiat ; quelques années plus tard, la famille possède un parc automobile de trois véhicules, dont la Mercedes de Kamila. Le courtage, la spéculation foncière et immobilière leur permettent d'acheter un petit immeuble de trois étages place Higaz, à Héliopolis. En 1987, ils acquièrent un terrain, également à Héliopolis, et y font construire « l'immeuble de la mort » : il comportait 14 étages, dont 6 illégaux. Un an plus tard, ils (ont construire à Madinat Nasr un autre immeuble, qui abrite le siège de la société immobilière enregistrée au nom de Kamila ainsi qu'un grand restaurant au rez-de-chaussée, lieu de rendez-vous des notabilités qui couvrent les activités illicites de Kamila et de sa famille... Ainsi cette «dame de fer » réussira-telle, par différents subterfuges, à échapper au paiement de près d'un million de LE d'amendes pour infractions aux règlements de la construction! (Rose at-Youssef, 26/10)

La campagne de presse contre «les assassins d'Héliopolis» est allée plus loin en dévoilant l'existence de dizaines de "hajj» (on appelle ainsi les musulmans ayant effectué le pèlerinage à la Mecque) promoteurs millionnaires qui échappent à toute sanction en dépit des nombreux jugements prononcés à leur encontre. Le lobby qu'exerce ce groupe de promoteurs-entrepreneurs a réussi à mettre en échec la décision du gouverneur du Caire de geler les constructions dans la capitale pendant un an : leur contre-attaque dans la presse a fait revenir le gouverneur sur sa décision au bout de trois mois! Les arguments développés par certains journalistes à leur solde frisent l'humour noir: "Cette décision ne peut que faire flamber les prix des appartements, dans un contexte où la demande va forcément augmenter du fait de l'effondrement de nombreux immeubles » (...) «Il devient nécessaire d'encourager la construction pour répondre aux besoins des sans-abri » (al-Ahrâm, 27/10); «Je ne comprends pas la logique de cette décision, qui ne mènera pas à résoudre la crise du logement » (al-Ahrâm, 28/10). Tout se passe donc comme s'il n'existait pas 1,8 million d'unités d'habitation vacantes, comme si les sans-abri constituaient la clientèle cible des promoteurs et comme si ces derniers allaient, du jour au lendemain, contribuer à résoudre le problème du logement... 
Séisme et aménagement du territoire

Si les études de sismicité ont un aspect strictement scientifique en ce qu'elles tracent les principaux traits de la tectonique globale actuelle, elles ont aussi un caractère pratique, car elles permettent de délimiter les régions où des précautions doivent être prises pour assurer la protection de l'homme. La confrontation des cartes d'épicentres, des cartes d'intensités maximales observées pendant une période aussi longue que possible et des cartes sismo-tectoniques - rendant possible, en particulier, l'identification des failles actives - permet de tracer des cartes d'intensités maximales généralisées fournissant à l'ingénieur et à l'architecte la documentation de base pour le calcul de normes parasismiques.

Ces études préalables auraient dû être effectuées pour orienter le choix des sites des villes nouvelles en Égypte. Or on a constaté, après le séisme du 12/10, que ce choix a été des plus hasardeux, et que de nombreux sites comportent des failles qui risquent de s'activer à tout moment. Tel est le cas des villes nouvelles du Six-Octobre et du QuinzeMai, comme l'affirme M. Megahed, assistant à l'Institut national des Sciences astronomiques et géographiques : « La ville du Quinze-Mai est construite sur un terrain qui comporte huit failles, dont la plus petite s'étend sur deux kilomètres de long et autant en profondeur... Ces failles risquent de s'activer du fait de l'existence, dans les environs, de cinq carrières qui utilisent la dynamite pour faire éclater les rochers. Une grande partie de l'énergie qui se dégage de ces explosions pénètre en profondeur et augmente la pression interstitielle dans les couches poreuses, au voisinage d'un accident tectonique jusque-là inactif... Rappelons qu'il existe quinze carrières dans la région du Fayoum. » (al-Ahrâm al-Masâ'i, 16/10) Le choix des sites des villes nouvelles du Fayoum, d'Assouan et de Tiba al-Gadida (Luxor) a été remis en question pour des raisons du même ordre. Le GOPP (organisme central de planification nationale et régionale) a entamé des études géophysiques visant à exclure de la construction les sites à hauts risques. Ces études, dont le coût est estimé à 200.000 LE, pourraient conduire à annuler des décisions déjà arrêtées, cela même si les travaux ont déjà commencé comme c'est le cas pour la ville nouvelle du Fayoum (al-Gumhuriyya, 21/11).

Ont également été abordés, outre la question du choix des sites des villes nouvelles, certains aspects de la crise relatifs aux politiques urbaines. M. al-Husayni a mis en évidence les contradictions de la politique du logement: «On n'a pas besoin d'être spécialiste de la politique du logement pour constater qu'au moment où des centaines de ménages vivaient dans des logements vétustes, une part importante des investissements étaient affectés à l'édification de stations balnéaires le long de la côte nord, entre Alexandrie et Marsa Matrouh... » (al-Musawwar, 30/10) L'extension de la mégapole par l'implantation de cinq villes nouvelles et satellites, en plus des dix new settlement dans la région du Grand Caire, inquiète le géologue A. Noweir, selon lequel une telle concentration démographique risque d'augmenter considérablement le nombre de victimes en cas d'évacuation. Il en conclut que «la construction d'une nouvelle capitale à l'extérieur du Delta et de la vallée » apparaît dès lors " inéluctable » (al-Siyâsi al-Masri, 25/10).

111 L'idée de déplacer la capitale de l'Égypte dans le désert, qui ressort aux moments des crises, n'a pas trouvé d'écho. Ce sont surtout les questions de rénovation et de restauration du patrimoine qui ont donné lieu à une pléthore de déclarations d'intentions "incohérentes », " contradictoires », " paradoxales ", " démesurées » et « irrationnelles », selon les termes de A. Ibrahim, directeur du Centre d'études urbaines 
et architecturales, qui estime que «chaque responsable décide de l'avenir du Caire historique comme s'il avait le pouvoir absolu et sans coordination avec les autres... Ainsi le gouverneur de la capitale a-t-il annoncé l'installation de commerces sous l'auto-pont de la rue al-Azhar, au moment où le ministre de la Culture annonçait un projet de « halles » couvrant une superficie de $5.000 \mathrm{~m} 2$ à Darrasa, destinées à accueillir les petits commerces déplacés de la rue al-Muiz! Projet qui vise à décongestionner la vieille ville et qui contredit totalement le premier. Quant au ministre de l'Habitat, il a dévoilé son dessein d'évacuer de toutes les zones historiques les activités polluantes leur portant atteinte, en application d'un projet global de rénovation urbaine qui aurait recours à des experts espagnols, italiens, Japonais et français... On lit ensuite dans la presse les grandes lignes d'un projet de réaménagement du nord de Gamaliyya, géré et finance par la France qui se déclare prête à y affecter 50 millions FF. Or, un projet similaire préparé par un bureau de consultants égyptiens sommeille depuis 1985 dans les tiroirs du gouvernorat... D'autre part est décidée la création d'une société égyptosaoudienne de promotion foncière et immobilière dotée d'un capital de 100 millions $\$$ qui aurait pour objectif d'acquérir et d'aménager, au Caire et dans d'autres villes égyptiennes, des terrains débarrassés de leurs décombres après effondrement des immeubles qui y existaient »... Poursuivant sa polémique en alignant les contradictions "qui se sont manifestées plus que jamais après le séisme", A. Ibrahim déplore " l'absence chronique d'une vue d'ensemble capable de définir les priorités et d'opérer des choix pertinents en matière d'aménagement urbain " (al-Ahrâm al-lqtisâd'i, 23/11).

Dans les mois qui ont suivi le séisme, la presse a continué à débattre, quoiqu' avec moins de passion, des effets de la catastrophe et des mesures à adopter, mettant plus particulièrement l'accent sur le nouveau code de construction et ses règlements. Comme on tentait d'élaborer le meilleur code possible, un immeuble endommagé par le séisme s'est effondré à Héliopolis le 3 mars 1993, causant autant de morts que l'effondrement du premier immeuble en octobre. L'expertise a conclu à des erreurs fatales de restauration.

Le dossier des défaillances du secteur du bâtiment en Égypte demeure ouvert.

Textes traduits et commentés par G. El Kadi

\section{ANNEXES}

Les grands tremblements de terre en Égypte

« L'Égypte n'a pas connu, tout au long de son histoire, de tremblement de terre d'une magnitude supérieure à 6,2 sur l'échelle de Richter. Le géologue Ruchdi Saïd estime que le séisme qui frappa l'Égypte en 1847, dont l'épicentre coïncide avec celui du 12 octobre 1992, peut être considéré comme le plus violent de l'histoire contemporaine de l'Égypte ; comme aujourd'hui, les villes les plus touchées furent Le Caire et la région du Fayoum: Le bilan de ce séisme parut dans le $n^{0} 84$ de la revue al-Waqâ'i' al-Masriyya (1847) : 68 habitations furent partiellement ou totalement détruites au Caire, 10 minarets perdirent leur bulbe, 6 arcades de la mosquée d'al-Mu'ayyad furent 
endommagées. Les pertes humaines se réduisaient à une seule victime. Mais c'est dans la région du Fayoum que les dégâts furent considérables :

2.987 maisons, 42 mosquées, un réservoir d'eau et 45 pigeonniers furent entièrement détruits. Les pertes humaines s'élevaient à 37 hommes et 48 femmes. 56 animaux domestiques avaient également péri. La Moyenne et la Haute-Égypte subirent également de grosses pertes.

Au cours de l'histoire, la Basse-Égypte a connu plusieurs séismes : en 2800 av. J.-C-, la ville de Bubastis fut détruite par un tremblement de terre d'une magnitude estimée à 7 ; la ville de Belbeis fut frappée en 859 et 865 par deux tremblements de terre assez violents ; en 1303, un séisme ayant son épicentre en Méditerranée frappa Damiette, Damanhur et Alexandrie, provoquant l'effondrement du célèbre phare d'Alexandrie ; trois autres séismes méditerranéens, survenus en 1856, 1926 et 1955, ont sérieusement affecté les villes de Rosette, Edkou, Damanhur, Mahmudiyya et Abu Homos. En HauteÉgypte, le séisme le plus connu date de 27 av. J.-C. ; il causa l'effondrement partiel des temples de Louxor et de Karnak. Quelques siècles plus tard. en 1801, un autre tremblement de terre s'y produisait.

En Nubie, un séisme a frappé la région d'Abu Simbel vers 1210 av. J.-C. Tout au long de l'époque romaine et jusqu'à nos jours, la grande ligne de fracture de Kalabcha, à $60 \mathrm{~km}$ au sud d'Assouan, s'est activée à plusieurs, reprises, provoquant des secousses dont la plus récente date de 1981. Ces secousses n'ont donc rien à voir avec le lac Nasser. » (alQâhira, décembre 1992)

Bilan des dégâts provoqués par le séisme dans chaque gouvernorat

Le Caire : 208 morts. 800 immeubles effondrés, 9.000 fissurés. 3 camps d'accueil aménagés sur le stade de la ville d'al-Salam, au Haikstep et à Helwan, pouvant héberger chacun 3.000 ménages.

Giza : 208 morts. 9 immeubles effondrés en milieu urbain. 3.500 maisons en milieu rural. 260 ménages relogés, 1.000 logements disponibles à al-Munib et dans la ville du Six-Octobre, ainsi que 220 logements à Imbaba.

Minufiyya ; 125 lycéennes blessées, 13 écoles effondrées et 432 autres fissurées. 302 avis de dommages dans divers édifices, dont le bâtiment de la direction des impôts, évacué. 580 unités de logements mis à la disposition des sinistrés.

Qaliubiyya : 58 morts, 338 blessés, 79 écoles hors d'usage, 4.300 appartements endommagés, 180 ménages relogés.

Fayoum : 46 morts, destruction totale de 3.700 maisons dans 3 villages, 27 bâtiments publics et 124 écoles fissurés ainsi que 100 mosquées et églises. 500 maisons partiellement ou totalement effondrées dans la ville de Fayoum.

Gharbiyya : 12 morts (écoliers) dont 11 à Mahalla al-Kubra et 1 à Kafr al-Zayat, 150 maisons fissurées ainsi que 60 centres de soins. Deux camps d'accueil d'une capacité de 40 ménages à Tanta et de 40 à Mahalla, aménagés pour héberger provisoirement les sans-abri.

Charqiyya : 3 morts dont deux lycéennes. 160 écoles hors d'usage et 50 fissurées. 2.465 maisons endommagées dont 191 évacuées. 500 unités de logement disponibles pour reloger les sinistrés. 
Bani Sweif : 5 morts, 11 maisons effondrées en milieu urbain et rural, 19 écoles fissurées, 60 ménages relogés. (October, 25/10)

Les zones sismiques en Égypte

Les foyers sismiques se concentrent dans trois cercles principaux :

- le premier est le cercle mer Rouge/Alexandrie. Il suit une crête sous-marine qui longe la mer Rouge, elle-même considérée comme un fossé fracturé, se prolonge ensuite vers le nord-ouest jusqu'à Alexandrie el la Méditerranée. Les foyers les plus actifs ont été localisés au mont Diab, à proximité de Qusayr, ainsi que dans l'île de Chadwan à l'entrée du Golfe de Suez ;

- le deuxième cercle constitue l'arc de Péluse/Fayoum/al-Galf al-Kabir. Il passe par les gouvernorats du Caire el de Charqiyya et se prolonge ensuite au nord-est vers la Palestine. Les foyers les plus actifs se trouvent au mont Qatrani, aux limites du lac. Ce cercle recoupe le premier aux environs du Caire ;

- le troisième cercle longe le golfe d'Aqaba el suit la direction du nord Jusqu'au Jourdain ; il est lié au fossé fracturé d'Aqaba et recoupe le premier cercle à proximité de l'île de Chadwan. (A. Noweir, al-Siyâsi al-Masri, 22/10)

Textes traduits de l'arabe par G. El Kadi et S. Rizq

INDEX

Mots-clés : Le Caire, logement, construction, crise, séisme, tremblement de terre

\section{AUTEUR}

GALILA EL KADI

ORSTOM / Faculty of Urban and Regional Planning, Cairo University 